\title{
Splitting of the Dipole and Spin-Dipole Resonances
}

\author{
Sam M. Austin ${ }^{1 *}$, E. Adamides ${ }^{1}$, A. Galonsky ${ }^{1}$, T. Nees ${ }^{1}$, W.A. Sterrenburg ${ }^{1 \dagger}$, \\ D.E. Bainum ${ }^{2 \ddagger}$, J. Rapaport ${ }^{3}$, E. Sugarbaker ${ }^{4 \S}$, C.C. Foster ${ }^{5}$, C.D. Goodman ${ }^{5}$, \\ D.J. Horen ${ }^{6 * *}$, C.A. Goulding ${ }^{7 \dagger \dagger}$, and M.B. Greenfield ${ }^{7 \ddagger}$ \\ ${ }^{1}$ National Superconducting Cyclotron Laboratory and Department of Physics and Astronomy \\ Michigan State University, East Lansing, MI 48824 \\ 2 Department of Physics, Emporia State University, Emporia, KS 66801 \\ 3 Department of Physics, Ohio University, Athens, OH 45701 \\ ${ }^{4}$ Department of Physics, University of Colorado, Boulder, CO 80309 \\ ${ }^{5}$ Indiana University Cyclotron Facility, Indiana University, Bloomington, IN 47405 \\ ${ }^{6}$ Oak Ridge National Laboratory, Oak Ridge, TN 37830 \\ ${ }^{7}$ Department of Physics, Florida A\&M University, Tallahassee, FL32307
}

(November 2, 2018)

\begin{abstract}
Cross sections for the ${ }^{90,92,94} \operatorname{Zr}(p, n)$ reactions were measured at energies of 79.2 and 119.4 MeV. A phenomenological model was developed to describe the
\end{abstract}

\footnotetext{
*Electronic address: austin@nscl.msu.edu

${ }^{\dagger}$ Present address: Dept. of Physics, Free University, 1007 MC Amsterdam, The Netherlands

${ }^{\ddagger}$ Present address: Washburn University, Topeka KS 66621

§Present address: Van de Graaff Lab., Ohio State University, Columbus, OH 43212

**Present address: 19 Colonial Ave., Framingham, MA 01701

${ }^{\dagger}$ Present address: LANL, MS J562 NIS-6, Los Alamos, New Mexico 87545

${ }_{\ddagger}$ Present address: Department of Physics, Int’l Christian University, Mitaka, Tokyo 181 Japan
} 
variation with bombarding energy of the position of the $L=1$ peak observed in these and other $(p, n)$ reactions. The model yields the splitting between the giant dipole and giant spin dipole resonances. Values of these splittings are obtained for isotopes of $\mathrm{Zr}$ and $\mathrm{Sn}$ and for ${ }^{208} \mathrm{~Pb}$.

PACS numbers: $25.40 . \mathrm{Kv}, 24.30 . \mathrm{Cz}$

Typeset using REVTEX 


\section{INTRODUCTION}

Inelastic scattering and charge exchange reactions induced by intense neutrino fluxes during supernova explosions play an important role in nucleosynthesis in supernovae. For example, they may produce rare light isotopes such as ${ }^{7} \mathrm{Li},{ }^{11} \mathrm{~B}$ and ${ }^{19} \mathrm{~F}$ [1] that are not sufficiently produced by the usual processes of nucleosynthesis and may modify the distribution of elements formed during the r-process [2]. Observation of supernova neutrinos in terrestrial detectors sensitive to neutrino flavor [3] may allow one to determine or limit the masses of the $\mu$ and $\tau$ neutrinos and to determine whether oscillations occur. From the point of view of nuclear physics, all these phenomena share a common ingredient: they depend on the $L=0$ allowed (Gamow Teller) and $L=1$ forbidden strengths of transitions induced by the various neutrino flavors. It may be possible to measure some of these strengths directly using neutrino beams, but it will be necessary to infer most of them from the cross sections for hadronic charge exchange and inelastic scattering reactions.

Unfortunately, information on the nature of the $L=1$ strength is not yet sufficiently detailed for such applications. The spectrum observed at low momentum transfer in a typical $(p, n)$ reaction is dominated by three isovector excitations: the isobaric analog state (IAS), the giant Gamow Teller Resonance (GTR), and the giant dipole $(L=1)$ resonance 46 . It is well known that the IAS contains almost all the Fermi strength and that the Gamow Teller peak contains about $60 \%$ of the sum rule value. Much less is known about the dipole peak and it exhibits a rather unusual behavior; it moves to lower excitation energy as the bombarding energy increases as shown in Fig. 1.

The qualitative reason for this behavior seems clear; the $L=1$ peak contains two separate structures: the giant dipole resonance (GDR) formed by a spin transfer of $S=0$, and the spin dipole resonance (SDR) formed by $S=1$. These resonances lie at different positions, but are sufficiently wide that they overlap. Because their relative excitation is expected to change in the energy range from 40 to $200 \mathrm{MeV}$, that of the SDR becoming relatively stronger at higher energy [7], the $L=1$ peak will shift toward the SDR as the energy 
increases. The amount of the shift depends on the energy splitting of the GDR and SDR; it should then be possible to obtain an estimate of this splitting from an analysis of the observed shifts.

The $\mathrm{Zr}$ isotopes are an excellent place to study the shift phenomenon. There have been $(p, n)$ studies on ${ }^{90} \mathrm{Zr}$ [8 [1] at 41, 45, 120, and $200 \mathrm{MeV}$, and on other Zr isotopes at $45 \mathrm{MeV}$

[9] and $200 \mathrm{MeV}$ [1]. This paper describes measurements of the ${ }^{90,92,94} \mathrm{Zr}(p, n)$ reactions at two intermediate energies, 79.2 and $119.4 \mathrm{MeV}$; a model that can account for the energy variation observed for the most complete data set $\left({ }^{90} \mathrm{Zr}\right)$; and the application of this model to extract the splitting of the $S=0$ and $S=1$ components of the $L=1$ resonance for several other nuclei. The basic assumptions of this model are similar to those employed by Taddeucci et al. [12] in the description of $L=0$ charge exchange reactions with $S=0$ and $S=1$.

The model also provides an improved estimate of the position of the GDR obtained from $(p, n)$ reaction studies. In earlier work it has simply been assumed that at bombarding energies near $50 \mathrm{MeV}$ the observed excitation is dominated by the GDR; we show that this is not the case. We note that one cannot simply obtain the position of the GDR in the charge exchange channel from photonuclear studies on the target nucleus. In nuclei with substantial values of $\mathrm{N}-\mathrm{Z}$, the dominant excitation in $(p, n)$ is the $T-1$ component of the GDR where $\mathrm{T}$ is the isospin of the target nucleus. The collective shift of this resonance differs from those of the $T$ and $T+1$ components seen in the target. Phenomenologically, this is accounted for by an isotensor component of the effective isospin potential [9, 13], which is poorly known. Consequently, the location of the $T-1$ GDR must be taken as unknown.

\section{EXPERIMENTAL PROCEDURES AND RESULTS}

Data were taken using the beam swinger time of flight facility at the Indiana University Cyclotron Facility [14], with two $15 \mathrm{~cm} \times 15 \mathrm{~cm} \times 100 \mathrm{~cm}$ detectors, located $46.5 \mathrm{~m}$ and $70.15 \mathrm{~m}$ from the target. Bombarding energies were $79.2 \mathrm{MeV}$ and $119.4 \mathrm{MeV}$, nominally 
80 and $120 \mathrm{MeV}$. Targets were enriched ${ }^{90,92,94} \mathrm{Zr}$ metal with thicknesses of 77.5, 25.4, and $41.6 \mathrm{mg} / \mathrm{cm}^{2}$, respectively. The overall resolution was between 415 and $540 \mathrm{keV}$ (580 and $675 \mathrm{keV})$ at $79.2 \mathrm{MeV}(119.4 \mathrm{MeV})$, significantly smaller than the width of the $L=1$ peaks we are concerned with here. Spectra were taken at about 0.1, 4, 8, 12, 16 and $19.5 \mathrm{deg}(\operatorname{lab})$. Those for ${ }^{90} \mathrm{Zr}$ at $4 \mathrm{deg}$ are shown in Fig. 2; at this angle the IAS and the GT resonances, which peak at $0 \mathrm{deg}$, and the $L=1$ resonances, which peak at 7-8 deg are visible.

Spectra were fitted with sums of Gaussian peaks plus a quadratic background, as shown in Fig. 2. The resulting fits are satisfactory. For angles of 4 deg and greater a single gaussian provides a good description of the $L=1$ strength. This is somewhat surprising because the $S=0, S=1$ splitting is expected to be in the range of 3.0 to $4.5 \mathrm{MeV}$ [15], not so much smaller than the width of the GDR, and because the relative contributions of the GDR and SDR are expected to change from 1:1 to about 1:20 over the energy range studied.

A numerical experiment was performed to evaluate the uncertainties that might arise from this procedure. Gaussian peaks with widths of $4.5 \mathrm{MeV}$ (representing the GDR) and $8 \mathrm{MeV}$ (representing the SDR), and $3.9 \mathrm{MeV}$ apart, were added and their sum was fitted by a single Gaussian. This was done for peak ratios from 1:1 to 1:20. In no case did the centroid of the fitted Gaussian differ from the actual centroid by more that $0.15 \mathrm{MeV}$, and most differences were much smaller. In another test, the centroid of the counts above the fitted background was calculated and compared to the centroid of the fitted Gaussian. The difference was $0.17 \mathrm{MeV}$. This gives one some confidence in the procedure followed at about the $\pm 0.1 \mathrm{MeV}$ level. The resulting energies for the dipole peak are shown in Fig. 1; the $L=1$ peak shifts to lower energies by about $2 \mathrm{MeV}$ as the bombarding energy changes from 40 to $200 \mathrm{MeV}$. The peak positions are consistent with centroid energies obtained by others at both lower and higher bombarding energies (see Fig. 4), lending some confidence to the procedures used here. 


\section{MODEL AND RESULTS}

We outline here a phenomenological model that describes the observed shift with bombarding energy in terms of the positions of the GDR and the SDR. The basic idea is as

outlined in the Introduction: that the energy of the $L=1$ peak moves toward the energy of the SDR as the bombarding energy increases. The notation we use is shown in Fig. 3. We denote the energy of the $S=0$ component, the $J^{\pi}=1^{-}$GDR, by $E_{0}$. Similarly, the energy of the $S=1$ component, the SDR consisting of $0^{-}, 1^{-}$, and $2^{-}$states has a combined centroid denoted by $E_{1}$. The states with different $J$ will have different distributions of strength with excitation energy. We lack the information to treat these differences in excitation in more detail, but believe that the effects are probably small compared to other uncertainties in the model.

Because of the nature of the two-nucleon interaction mediating the transitions, the strength of the $S=0$ excitation of the GDR is comparable to that of the $S=1$ excitation of the SDR at $45 \mathrm{MeV}$, but $S=1$ becomes much stronger than $S=0$ as the bombarding energy increases toward $200 \mathrm{MeV}$. One then expects the observed centroid of the $L=1$ strength to move toward $E_{1}$ with increasing bombarding energy. Rather general arguments 15] indicate that the $S=0$ GDR lies at higher excitation energy than the centroid of the $S=1$ SDR so this motion corresponds to a decreasing excitation energy. Detailed theoretical calculations [7] qualitatively confirm this expectation. See Refs. [4 6] for general discussion and references.

The position, $C$, of the centroid of the $L=1$ excitations (including both the GDR and SDR) at a bombarding energy $E_{p}$ is given by

$$
C=\frac{\sigma_{0} E_{0}+\sigma_{1} E_{1}}{\sigma_{0}+\sigma_{1}}=E_{0}-\frac{\sigma_{1} / \sigma_{0}}{1+\sigma_{1} / \sigma_{0}} \Delta
$$

where $\Delta=E_{0}-E_{1}$ and $\sigma_{0}\left(\sigma_{1}\right)$ is the cross section for $S=0(S=1)$ transfer. One can express $C$ in terms of $E_{0}$ and $\Delta$ if one knows the ratio $\sigma_{1} / \sigma_{0}$. We assume here that for the $L=1$ excitations this ratio is directly proportional to the ratio of the forbidden $S=1$ and $S=0$ 
beta decay strengths, as is observed for the $L=0$ case [12 where the analogous excitations are the Gamow Teller and IAS resonances. This assumption is confirmed for $L=1$ by the DWIA calculations of Gaarde et al. [16]. Then for small momentum transfers one has [12]

$$
\sigma_{1} / \sigma_{0} \approx \frac{K_{\sigma \tau}}{K_{\tau}} N_{D}^{1}\left|\frac{J_{\sigma \tau}}{J_{\tau}}\right|^{2} \frac{B(\mathrm{SDR})}{B(\mathrm{GDR})},
$$

where $J_{\sigma \tau}$ and $J_{\tau}$ are the volume integrals of the parts of the effective interaction mediating $S=1$ and $S=0$ transitions respectively, the $B$ 's are the corresponding transition strengths and the $K$ 's are kinematic factors whose ratio is very near 1.0 for our case. $N_{D}^{1}$ $=\left(N_{\sigma \tau} / N_{\tau}\right)_{L=1}$ where the $N$ 's are distortion factors which differ from each other by about $15 \%$ at the first peak in the $L=1$ angular distribution. For $L=0$ transitions $N_{D}^{0}\left|J_{\sigma \tau} / J_{\tau}\right|^{2}$ $=a^{2} E_{p}^{2}$ where $N_{D}^{0}=\left(N_{\sigma \tau} / N_{\tau}\right)_{L=0}, a=(55 \mathrm{MeV})^{-1}$ and $E_{p}$ is the bombarding energy [12]. This relationship is accurate over the energy range of 45 to $200 \mathrm{MeV}$ studied here. One then obtains

$$
C=E_{0}-\frac{\alpha a^{2} E_{p}^{2}}{1+\alpha a^{2} E_{p}^{2}} \Delta .
$$

Here $\alpha$ is given by $\left(N_{D}^{1} / N_{D}^{0}\right)[B(\mathrm{SDR}) / B(\mathrm{GDR})]$. In principle, experimental results can be used to fix $\alpha$ if data at three energies are available (there are three parameters $\Delta, E_{0}$ and $\alpha$ ). Fitting the ${ }^{90} \mathrm{Zr}$ results yields a value of $\alpha$ near 1 , but with a large uncertainty due to the limited accuracy of the centroid data. It is then desirable to obtain an estimate of $\alpha$ at least partly from other considerations.

The ratio of the $B$ 's depends on both the sum rule strengths for the transitions and the fraction $f$ of those strengths (quenching factor $=f$ ) found in the low excitation giant resonance peaks of interest here. Estimates with uncorrelated and with RPA ground states [17] give $B(\mathrm{SDR}) / B(\mathrm{GDR})=3.25$ and 3.2, respectively. Gaarde, et al. [16] have also shown that the effect of RPA correlations is small. For the $S=1$ quenching factor we take $f \approx$ 0.5, comparable to that obtained experimentally for the $L=0$ GT resonance [4]. A value of $f$ in the range of 0.3 to 0.5 has been observed by Gaarde et al. [16 for masses of 40 to 208. Several authors, e.g. Drożdż et al. [18, have shown that perhaps $30 \%$ of the total 
$L=1$ strength is moved to high energies. Strength at higher excitation has also been seen in polarization transfer experiments and in multipole decompositions of $(p, n)$ and $(n, p)$ data [4,6]. Our results then apply to the resonant part of the cross section, not that part lying higher in the continuum. Implicitly the model assumes that the largest concentration of strength, the peak in the excitation energy distribution for $L=1$, gives a reasonable estimate of the position of the collective strength for comparison with models. There is some indication in Ref. [18] that the position of maximum strength does not change greatly when continuum effects are considered. For $S=0$ the quenching factor is presumed to be near 1.0, in analogy with the $L=0, S=0$ (Fermi transition) case.

Finally $N_{D}^{1} / N_{D}^{0}$ is obtained from the DWIA calculations evaluated at the peaks of the relevant angular distributions (e.g. near $4.5^{\circ}$ for $L=1$ and at $0^{\circ}$ for $L=0$ at $200 \mathrm{MeV}$ on $\mathrm{Pb}$ ) and typically has a value near 1.0. Based on all these considerations we take $\alpha=1.5$ \pm 0.5 as covering the values of interest.

Fitting Eq. 3 with $\alpha=1.5 \pm 0.5$ to the data for ${ }^{90} \mathrm{Zr}$ yields the results shown in Fig. 4 . Similar procedures were followed for other isotopes for which data are available at widely separated bombarding energies. For ${ }^{92,94} \mathrm{Zr}$ the excitation energy data are shown in Fig. 1; for ${ }^{112,116,124} \mathrm{Sn}$ data are available at 45 and $200 \mathrm{MeV}$ [9,[1]]; and for ${ }^{208} \mathrm{~Pb}$ data are available at 41,45,120 and $200 \mathrm{MeV}$ [8,9,11, 19]. The results are shown in Table I. Also shown in Table I is the position of the GDR measured in pion induced charge exchange reactions. These data are not in good agreement with the $(p, n)$ results for ${ }^{90} \mathrm{Zr}$. However, the difference between the results of single and double charge exchange reactions for ${ }^{208} \mathrm{~Pb}$ indicates that there may be systematic uncertainties not yet accounted for.

Krmpotić, Nakayama and Pio Galeão [15] and Auerbach and Klein [20] have discussed the nature of the giant first forbidden resonances and have given values for the energies of the GDR and SDR: their results are given in Table I. They are in qualitative agreement with the results of a detailed calculation by Bertsch, Cha, and Toki [21] which places the centroid of the SDR near $18 \mathrm{MeV}$, below the GDR. Theory and experiment agree closely for ${ }^{208} \mathrm{~Pb}$ and within the uncertainties for the $\mathrm{Zr}$ isotopes, although the theoretical results are 
smaller. The agreement is less good for the Sn isotopes, perhaps because experimental data are available only at 45 and $200 \mathrm{MeV}$. It is difficult to estimate the theoretical uncertainties, but they could be a few MeV, mainly in the position of the spin-dipole excitation [22].

\section{DISCUSSION AND SUMMARY}

It is perhaps surprising that the the experimental and theoretical values of $E_{0}$ and $\Delta$ shown in Table I are in reasonably good agreement, since the experimental results do not measure the position of the high-lying continuum strength and the theoretical estimates include all strength. We do not have an explanation for this result, except possibly that the position of maximum strength calculated in RPA does not change much when continuum effects are included; see Ref. [18]. Calculations of the positions of the resonance peaks would be more appropriately compared with the present experiments.

Information similar to that in Table I can in principle be obtained from a comparison of the positions of the SDR observed in high energy $(p, n)$ reactions and the GDR observed in pion charge exchange; however, the energy shift approach outlined here at the least provides an independent measure of the GDR-SDR splitting, and arguably is less subject to systematic uncertainties arising from different experimental approaches. The present procedure is based on simple assumptions and simple Gaussian shapes for the dipole resonances. The procedure could be greatly improved if data of sufficient statistics and angular range were available at several energies for each target so that subtraction or multipole fitting procedures could be used to isolate $L=1$ strength. With better data, it would become worthwhile to model corrections owing to the (relatively small) contributions of the higher isospin $(T)$ components of the excitation; here we have implicitly neglected all but the $T=T_{0}-1$ components.

Even in its present form the model provides a quantitative explanation of the shift of the $L=1$ excitation with bombarding energy, a measure of splitting of the spin-dipole and dipole resonances, and an improved estimate of the position of the GDR peak (see Table I 
and Fig. 4). The results appear in a general way to support the assumptions made by Fuller et al. 3] in their calculations of the the response of a flavor-dependent supernova neutrino detector. They assume that the spin-dipole resonance does not lie at higher excitation energy than the GDR, in agreement with our finding. However, the present results only apply to the part of the strength that is in the resonant peaks, while the response of the detector will depend also on the part of the strength that lies in the continuum at higher $E_{x}$. In addition, it will be necessary to use a model to relate the observed splitting in the charge exchange channel to that in ${ }^{208} \mathrm{~Pb}$.

The results also indicate that past estimates of tensor contributions to the isospin splitting of the giant dipole resonance (e.g. Sterrenburg et al. [9]) must be modified. These estimates assumed that the GDR was at the position of the $L=1$ peak observed for bombarding energies near $50 \mathrm{MeV}$. Our results show that it lies at higher $E_{x}$, reducing the values of $E_{-}=E_{T}-E_{T-1}$ obtained in that paper.

To summarize, cross sections for the ${ }^{90,92,94} \operatorname{Zr}(p, n)$ reaction have been measured at energies of 79.2 and $119.4 \mathrm{MeV}$. The positions of the observed $L=1$ excitation have been extracted and compared with those at other energies; the excitation strength is observed to move toward lower excitation energy as the bombarding energy increases. A simple phenomenological picture was developed to describe the observed energy variation and was used to extract the splitting of the giant dipole (GDR) and giant spin-dipole (SDR) resonances. The results are in agreement with theoretical models as to the scale of the splitting, but the value of $\Delta$ we obtain is usually somewhat larger than predicted.

We have had valuable conversations with N. Auerbach, G. Bertsch, D.M. Brink, K. Nakayama and H. Toki. E. Adamides acknowledges the financial support of the Academy of Athens-Greece from the V. Notara Bequest. This research was supported by the National Science Foundation and the Department of Energy. 


\section{REFERENCES}

[1] S. E. Woosley, D. H. Hartman, and W. C. Haxton, Astrophys. J. 356, 272 (1990).

[2] W. C. Haxton, K. Langanke, Y.-Z. Qian, and P. Vogel, Phys. Rev. C 55, 1532 (1997).

[3] G. M. Fuller, W. C. Haxton, and G. C. McLaughlin, Phys. Rev. D 59, 085005 (1999).

[4] F. Osterfeld, Rev. Mod. Phys. 64, 491 (1992).

[5] J. Rapaport and E. Sugarbaker, Ann. Rev. Nucl. Part. Sci. 44, 109 (1994).

[6] W. P. Alford and B. M. Spicer, Adv. Nucl. Phys. 24, 1 (1998).

[7] F. Osterfeld, S. Krewald, H. Dermawan, and J. Speth, Phys. Lett. 105B, 257 (1981).

[8] S. Nishihara, K. Furukawa, M. Kabasawa, T. Nakagawa, H. Orihara, K. Maeda, K. Muira, and H. Ohnuma, Phys. Lett. 160B, 369 (1985).

[9] W. A. Sterrenburg, Sam M. Austin, R. P. DeVito, and A. Galonsky, Phys. Rev. Lett. 45, 1839 (1980).

[10] D. E. Bainum, J. Rapaport, C. D. Goodman, D. J. Horen, C. C. Foster, M. B. Greenfield, and C. A. Goulding, Phys. Rev. Lett. 44, 1751 (1980).

[11] D. J. Horen, C. D. Goodman, D. E. Bainum, C. C. Foster, C. A. Goulding, M. B. Greenfield, J. Rapaport, T. N. Taddeuchi, E. Sugarbaker, T. Masterson, S. M. Austin, A. Galonsky, and W. Sterrenburg, Phys. Lett. 99B, 383 (1981).

[12] T. N. Taddeucci, C. A. Goulding, T. A. Carey, R. C. Byrd, C. D. Goodman, C. Gaarde, J. Larsen, D. Horen, J. Rapaport, and E. Sugarbaker, Nucl. Phys. A469, 125 (1987).

[13] R. Leonardi, Phys. Rev. Lett. 28, 836 (1972).

[14] C. D. Goodman, et al., IEEE Trans. Nucl. Sci. 26, 2248 (1979).

[15] F. Krmpotić, K. Nakayama, and A. Pio Galeão, Nucl. Phys. A399, 478 (1983). 
[16] C. Gaarde, J. Rapaport, T. N. Taddeucci, C. D. Goodman, C. C. Foster, D. E. Bainum, C. A. Goulding, M. B. Greenfield, D. J. Horen, and E. Sugarbaker, Nucl. Phys. A369, $258(1981)$.

[17] K. Nakayama and H. Toki, (private communications).

[18] S. Drożdż, F. Osterfeld, J. Speth, and J. Wambach, Phys. Lett. 189B, 271 (1987).

[19] D. J. Horen, C. D. Goodman, C. A. Goulding, M. B. Greenfiedd, J. Rapaport, D. E. Bainum, E. Sugarbaker, T. G. Masterson, F. Petrovich and W. G. Love, Phys. Lett. 95B, 27 (1980).

[20] N. Auerbach and A. Klein, Nucl. Phys. A395, 77 (1983).

[21] G. Bertsch, D. Cha, and H. Toki, Phys. Rev. C 24, 533 (1981).

[22] N. Auerbach, private communication

[23] T. N. Taddeucci, C. D. Goodman, R. C. Byrd, I. J. Van Heerden, T. A. Carey, D. J. Horen, J. S. Larson, C. Gaarde, J. Rapaport, T. P. Welch, and E. Sugarbaker, Phys. Rev. C 33, 747 (1986).

[24] A. Erell, J. Alster, J. Lichtenstadt, M. A. Moinester, J. D. Bowman, M. D. Cooper, F. Irom, H.S. Matis, E. Piatetzky, and U. Sennhauser, Phys. Rev. C 34, 1822 (1986).

[25] R. A. Loveman, B. L. Clausen, R. J. Peterson, S. H. Rokni, H. W. Baer, A. G. Bergmann, J. D. Bowman, F. Irom, C. J. Seftor, J. Alster, E. Piasetzky, J. N. Knudson, and U. Sennhauser, Phys. Rev. C 40, 2710 (1989).

[26] S. Mordechai, N. Auerbach, S. Greene, C. L. Morris, J. M. O’Donnell, H. T. Fortune, G. Liu, M. Burlein, A. Wuosmaa, S. H. Yoo, and C. Fred Moore, Phys. Rev. C 40, 850 (1989). 


\section{FIGURES}

FIG. 1. Energies of the $L=1$ resonance for the Zr isotopes measured from the IAS; values are from the present work, and from Refs. [8 11]. The uncertainties of the present data include an estimate of the systematic errors; this also appears to be the case for the data from the other references.

FIG. 2. Spectra from ${ }^{90} \operatorname{Zr}(p, n)$ at 79.2 and $119.4 \mathrm{MeV}$, with background used in the analysis. The energy is measured from the IAS.

FIG. 3. Diagram showing assumed model and definition of terms. The zero of the energy scale is the energy of the IAS.

FIG. 4. Comparison of the $L=1$ resonance positions for ${ }^{90} \mathrm{Zr}$ and Eq. 3. Energies are measured from the IAS. The abscissa is the bombarding energy and the curves are non-linear least square fits to the data. 


\section{TABLES}

TABLE I. Energy ${ }^{\mathrm{a}}$ of the Dipole $\left(E_{0}\right)$ and of the Dipole-Spin-Dipole Splitting $(\Delta)$

\begin{tabular}{lccccc}
\hline \hline & \multicolumn{2}{c}{ Present $^{\mathrm{b}}$} & \multicolumn{2}{c}{ Theory $^{\mathrm{c}}$} & $\left(\pi^{+}, \pi^{0}\right),\left(\pi^{+}, \pi^{-}\right)$ \\
\hline Target & $E_{0}$ & $\Delta$ & $E_{0}$ & $\Delta$ & $E_{0}$ \\
& $\mathrm{MeV}$ & $\mathrm{MeV}$ & $\mathrm{MeV}$ & $\mathrm{MeV}$ & $\mathrm{MeV}$ \\
\hline${ }^{90} \mathrm{Zr}$ & $16.7 \pm 1.2(0.6)$ & $4.0 \pm 1.3(0.9)$ & $15.3,14.6$ & 3.0 & $14.2 \pm 0.5^{\mathrm{d}}$ \\
${ }^{92} \mathrm{Zr}$ & $17.1 \pm 1.6(0.8)$ & $5.1 \pm 1.7(1.1)$ & 15.0 & 3.4 & \\
${ }^{94} \mathrm{Zr}$ & $16.9 \pm 1.9(1.0)$ & $5.4 \pm 2.1(1.4)$ & 14.7 & 3.8 & \\
${ }^{112} \mathrm{Sn}$ & $16.6 \pm 2.1(1.0)$ & $6.8 \pm 2.7(1.7)$ & 13.8 & 2.8 & \\
${ }^{116} \mathrm{Sn}$ & $17.5 \pm 2.5(1.2)$ & $8.6 \pm 3.1(1.8)$ & 13.1 & 3.3 & \\
${ }^{124} \mathrm{Sn}$ & $14.4 \pm 2.2(1.0)$ & $7.5 \pm 2.8(1.7)$ & 12.3 & 4.3 & \\
${ }^{208} \mathrm{~Pb}$ & $11.0 \pm 1.5(0.8)$ & $4.7 \pm 2.0(1.4)$ & $10.3,9.9$ & 4.7 & $10.2 \pm 0.9^{\mathrm{e}}, 8.6 \pm 0.5^{\mathrm{f}}$ \\
\hline \hline
\end{tabular}

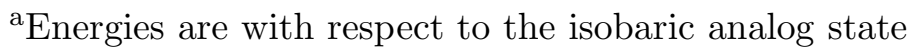

${ }^{\mathrm{b}}$ Calculated for $\alpha=1.5$. The uncertainties include an allowance for the uncertainty of \pm 0.5 in $\alpha$ : one half the difference in $E_{0}$ and $\Delta$ for fits with $\alpha=1.0$ and 2.0 is added linearly to the fit error. The first number following the \pm sign is the total error; the fit error is given in parentheses.

${ }^{\mathrm{c}}$ From Ref. [15], except the second values of $E_{0}$ for ${ }^{90} \mathrm{Zr}$ and ${ }^{208} \mathrm{~Pb}$ are from Ref. [20]

${ }^{\mathrm{d}}$ Mean of results in Refs. 24,25]

eRef. [26] $\left(\pi^{+}, \pi^{-}\right)$

${ }^{\mathrm{f}}$ Ref. [25], $\left(\pi^{+}, \pi^{0}\right)$ 


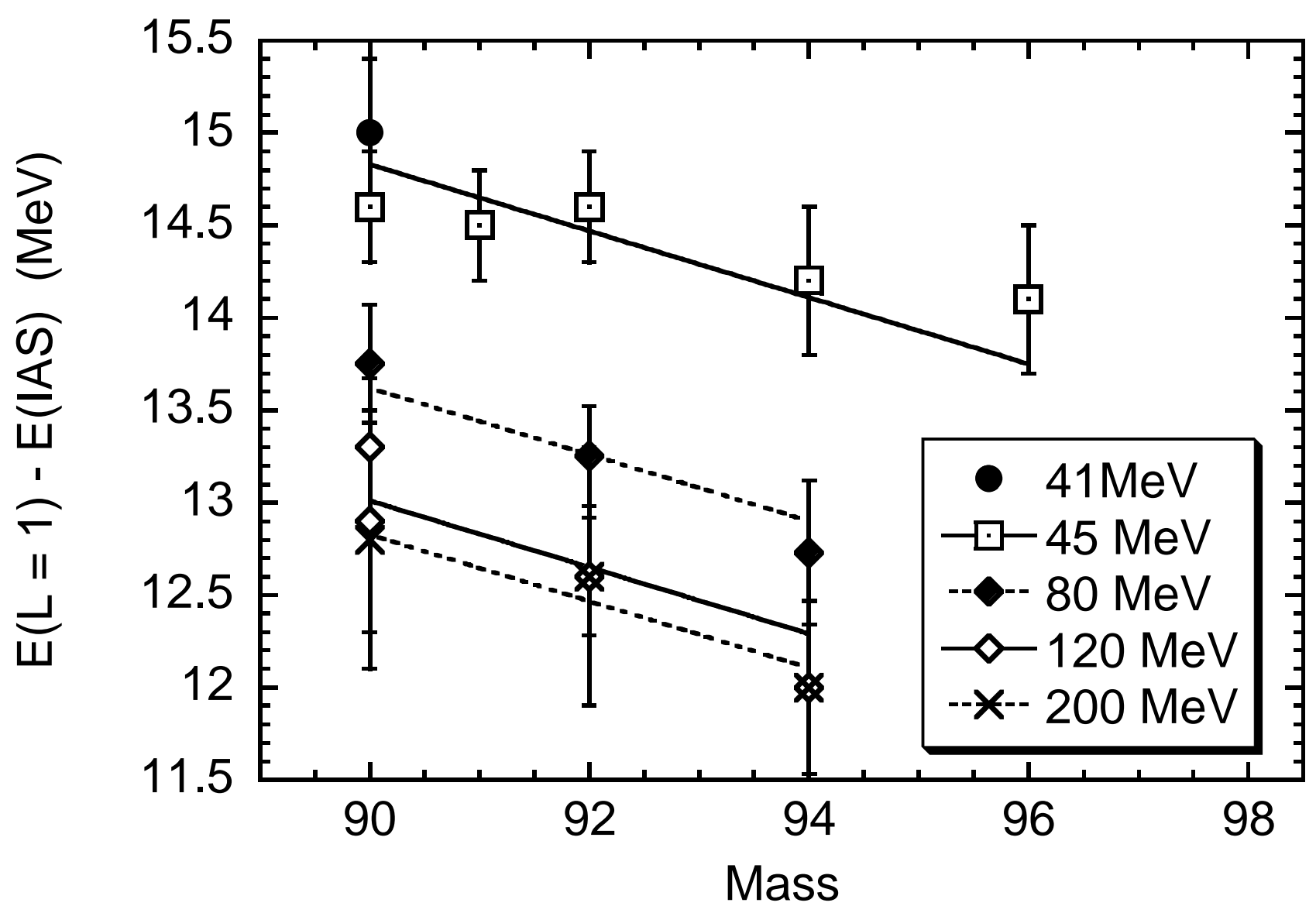



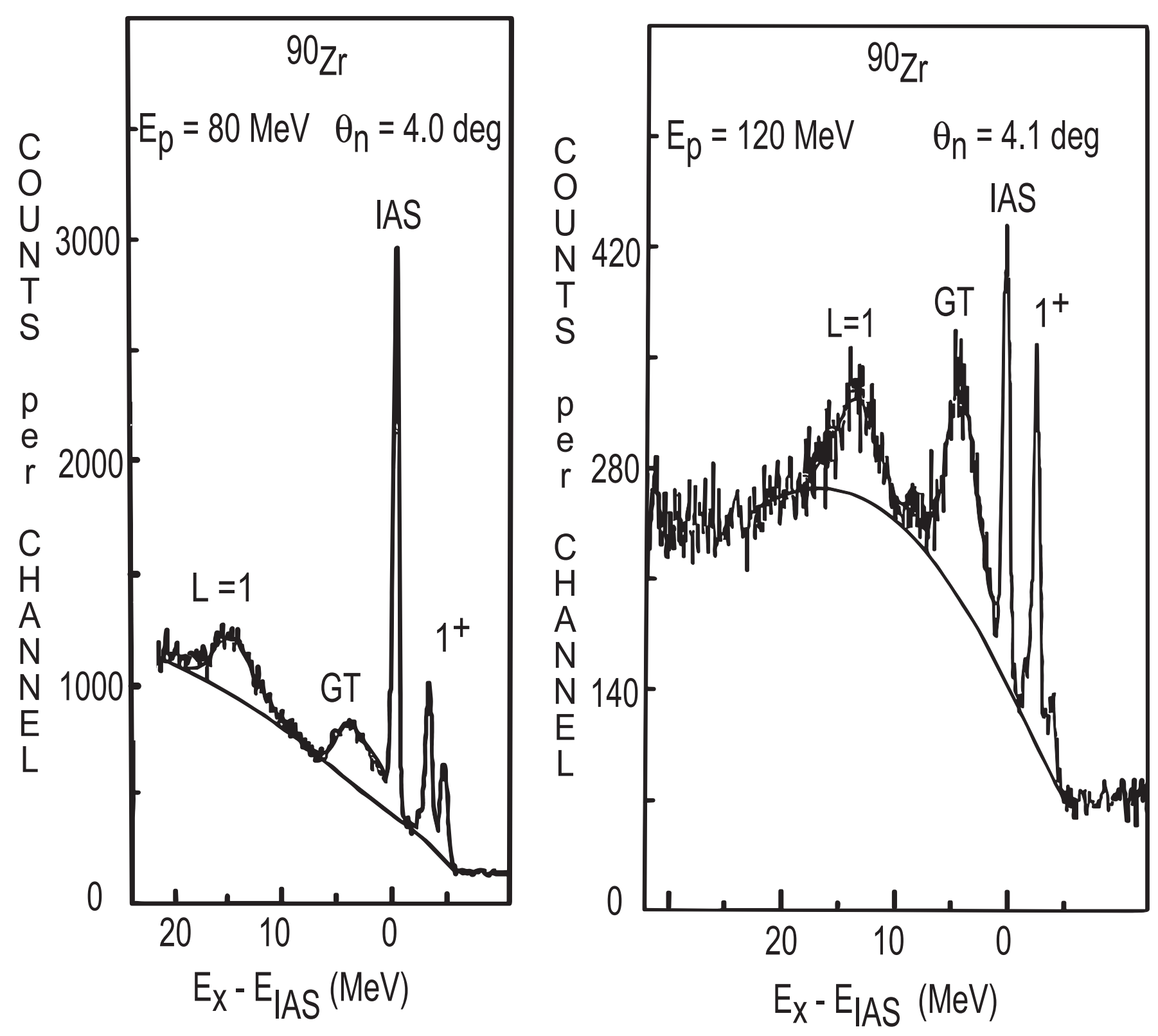


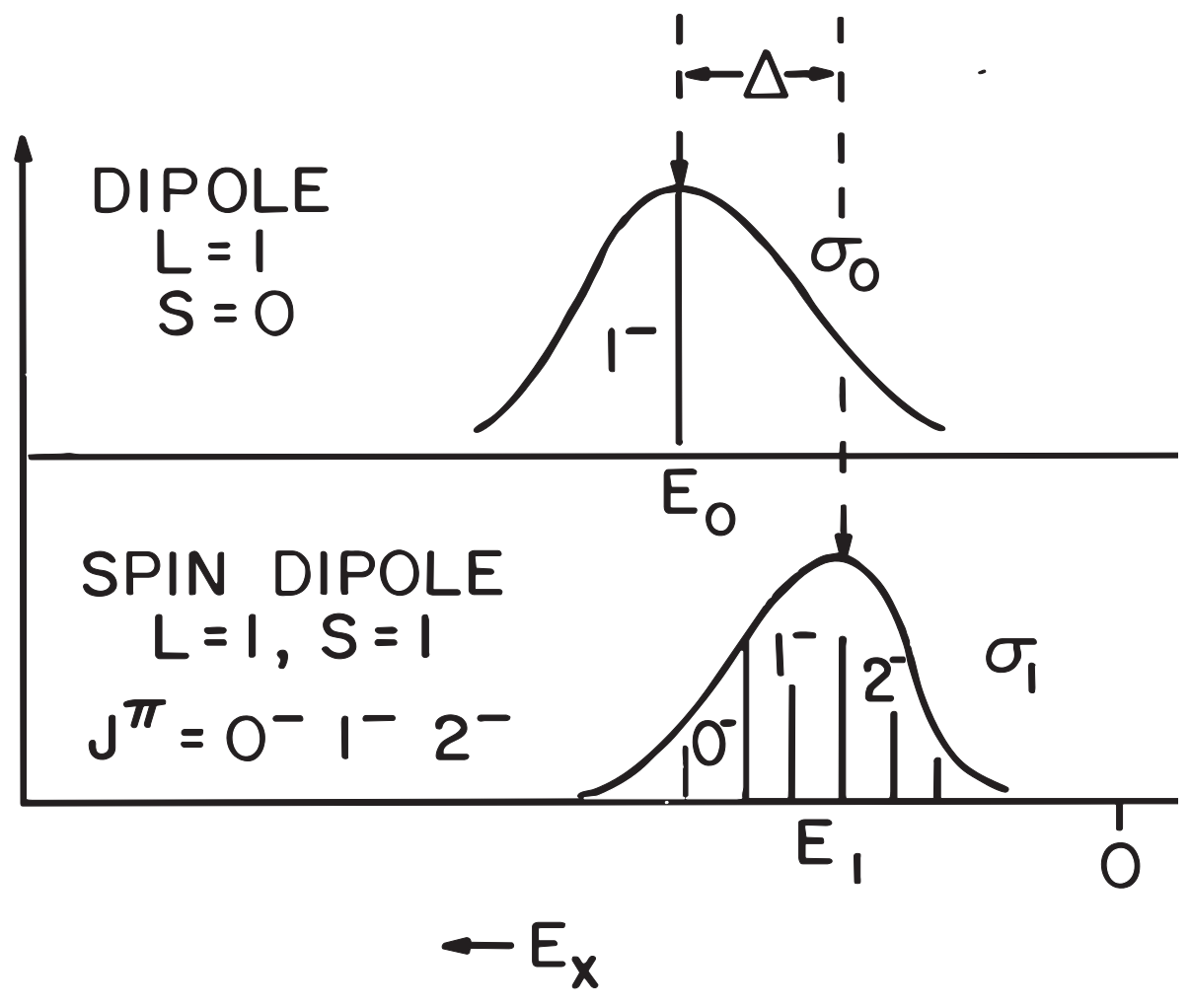




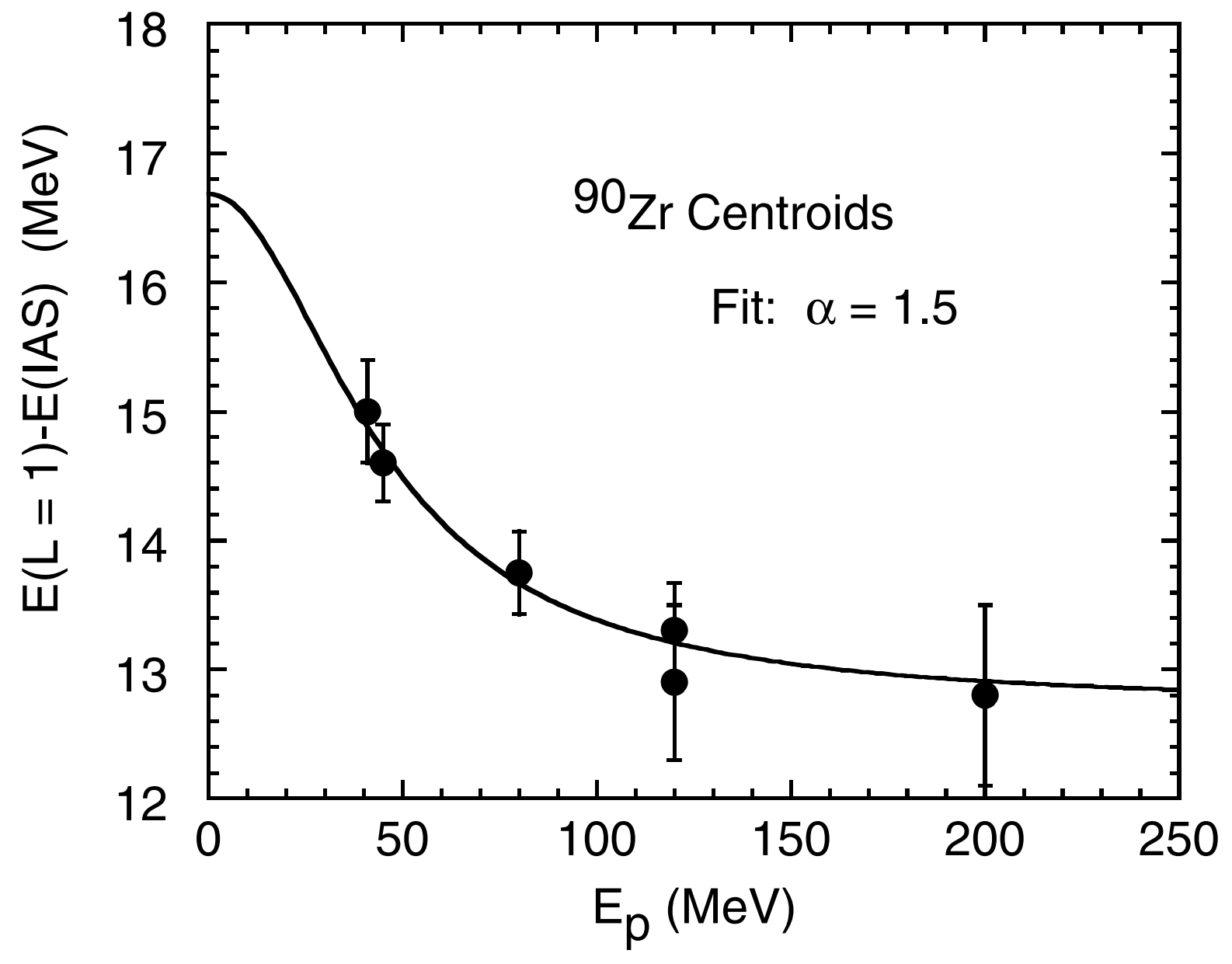

